\title{
The UCD Population of the Coma Cluster
}

\author{
Kristin Chiboucas ${ }^{1}$, Peter Ferguson ${ }^{1}$, R. Brent Tully ${ }^{2}$, David Carter ${ }^{3}$, \\ Steven Phillipps ${ }^{4}$ and Eric Peng ${ }^{5,6}$ \\ ${ }^{1}$ Gemini Observatory, Hilo, HI, United States \\ email: kchibouc@gemini.edu email: petersferguson@gmail.com \\ ${ }^{2}$ Institute for Astronomy, Honolulu, HI, United States \\ email:tully@ifa.hawaii.edu \\ ${ }^{3}$ Liverpool John Moores University, Liverpool, United Kingdom \\ email:d.carter@ljmu.ac.uk \\ ${ }^{4}$ University of Bristol, Bristol, United Kingdom \\ email:s.phillipps@bristol .ac.uk \\ ${ }^{5}$ Peking University, Beijing, China \\ ${ }^{6}$ Kavli Institute for Astronomy and Astrophysics, Beijing, China \\ email:peng@pku .edu.cn
}

\begin{abstract}
UCDs are super massive star clusters found largely in dense regions but have also been found around individual galaxies and in smaller groups. Their origin is still under debate but consensus is that they formed either during major galaxy mergers as mergers of super massive star clusters, are simply the high mass end of the globular cluster luminosity function and formed in the same way as globular clusters, or that they formed from the threshing of galaxies and are remnant nuclear star clusters, which themselves may have formed from the mergers of globular star clusters within galaxies. We are attempting to disentangle these competing formation scenarios with a large survey of UCDs in the Coma cluster. Using ACS two-passband imaging from the HST/ACS Coma Cluster Treasury Survey, we are using colors and sizes to identify the UCD cluster members. With a large sample within the core region of the Coma cluster, we will use the population size, properties, and spatial distribution, and comparison with the Coma globular cluster and nuclear star cluster populations to discriminate between the threshing and globular cluster scenarios. In particular, previously we have found a possible correlation of UCD colors with host galaxy and a possible excess of UCDs around a non-central giant galaxy with an unusually large globular cluster population, both suggestive of a globular cluster origin. With a larger sample size, we are investigating whether the color correlation with host persists and whether the UCD population is consistent with, or in excess of, the bright end of the GCLF. We present initial results from the survey.
\end{abstract}

Keywords. galaxies:star clusters - galaxies:dwarf - galaxies: clusters: individual (Coma)

\section{Methods and Results}

We have searched for UCD candidates in the 25 ACS fields centered on the core of the Coma cluster. Initial selection was based on the SExtractor FWHM, magnitude, half light radius, and $F 475 W-F 814 W$ measurements. These measurements for 34 spectroscopically confirmed UCDs in these fields were used to establish criteria for candidate selection. We are currently performing profile fitting on the initial candidates to measure sizes and shapes in order to refine the candidate list. With an accurately known PSF it is possible to measure effective radii for objects down to $\sim 10 \%$ of the PSF FWHM. For ACS imaging, we find we can measure sizes as small as $\sim 7 \mathrm{pc}$ at the distances of Coma, about the minimum size for UCDs. From the initial selection of candidates, we find an excess of UCDs around 3 prominent giants in the core region (see Figure 1) including 

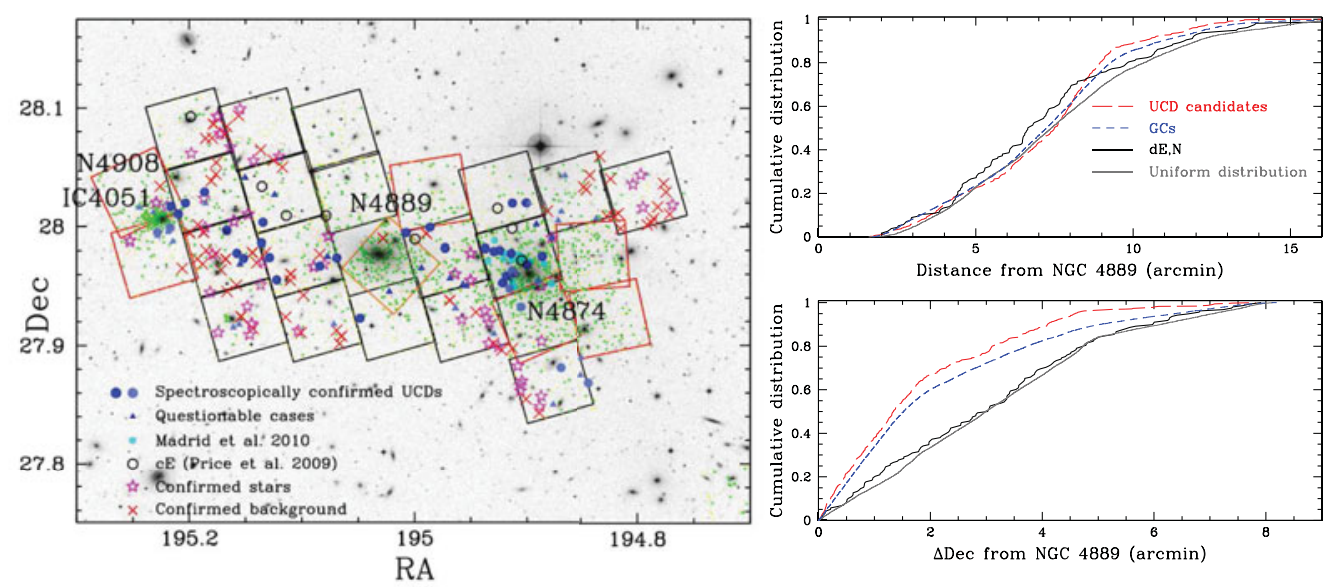

Figure 1. Left: Spatial distribution of UCDs in the Coma cluster. Large filled circles are spectroscopically confirmed. Small green points are UCD candidates chosen based on FWHM, surface brightness, color, and magnitude. Boxes indicate ACS fields from the HST/ACS Coma Cluster Treasury Survey (Carter et al. 2008), red boxes are more recent data. Right: Distribution as a function of distance from the most central cD galaxy NGC 4889 including only objects in the original fields. We find that globular clusters (Peng et al. 2011) and UCDs share a similar more concentrated distribution in declination with an excess in a band running between NGC 4874 and IC 4051 and around 3 giants in the core region. dEs exhibit a more uniform distribution.

around IC 4051, the same galaxy that hosts an unusually large globular cluster population. Overall, UCD candidates and the Coma cluster globular cluster population share a similar distribution. With properties and distribution more similar to Coma globular clusters than galaxies, we find more evidence for a star cluster origin for these objects. However, other origin scenarios are not yet ruled out.

\section{Future work}

From size and shape measurements of all candidates, we will determine probable cluster membership for UCDs with sizes $>7 \mathrm{pc}$ and establish a more complete sample of UCDs in the core region of the Coma cluster beyond the 34 spectroscopically confirmed members. In order to distinguish between different formation channels, we will investigate the spatial distribution in the cluster in comparison with the globular cluster and dwarf galaxy populations, determine the specific frequency of UCDs and GCs around the 3 major galaxies and analyze whether UCD counts are consistent with the bright tail of the globular cluster luminosity function, establish whether the color affiliations with host found previously are real, and compare the abundance of UCDs and dwarf galaxies in the core region to determine the efficiency of stripping required if UCDs are formed from the threshing of these galaxies.

\section{References}

Carter, D., Goudfrooij, P., Mobasher, B., et al. 2008 ApJS, 176, 424

Madrid, J. P., Graham, A. W., Harris, W. E., Goudfrooij, P., Forbes, D. A., Carter, D., Blakeslee, J. P., Spitler, L. R., Ferguson, H. C. 2010 ApJ, 722, 1707

Peng, E. W., Ferguson, H. C., Gudfrooij, P., Hammer, D., Lucey, J. R., Marzke, R. O., Puzia, T. H., Carter, D. 2011 ApJ, 730, 23

Price, J., Phillipps, S., Huxor, A., et al. 2009, MNRAS, 397, 1816 